\title{
Design, synthesis and preclinical evaluation of NRC-AN-019
}

\author{
KOMPELLA AMALA $^{1}$, A.K.S. BHUJANGA RAO ${ }^{1}$, BHARATHI GORANTLA ${ }^{2}$, \\ CHRISTOPHER S. GONDI ${ }^{2}$ and JASTI S. RAO ${ }^{2}$
}

\author{
${ }^{1}$ Natco Research Centre, Sanath Nagar, Hyderabad, India; ${ }^{2}$ Department of Cancer Biology and Pharmacology, \\ University of Illinois College of Medicine at Peoria, IL, USA
}

Received September 1, 2012; Accepted October 12, 2012

DOI: 10.3892/ijo.2012.1697

\begin{abstract}
Imatinib mesylate is the first tyrosine kinase inhibitor developed and approved for the treatment of chronic myeloid leukemia (CML). In the past few years development of resistance towards imatinib mesylate has been reported. To overcome this problem a series of phenyl amino pyrimidine derivatives have been designed, prepared and evaluated for antiproliferative activity against the BCR-ABL-positive leukemia cell line K562. Among these phenyl amino pyrimidine derivatives, NRC-AN-019 has been found to be a promising new lead compound for the therapy of imatinib mesylate-resistant chronic myeloid leukemia. In this communication, we describe the design, preparation and preclinical studies of NRC-AN-019.
\end{abstract}

\section{Introduction}

Chronic myeloid leukemia (CML) is characterized by the presence of the Philadelphia $(\mathrm{Ph})$ chromosome, which results from a reciprocal translocation between the long arms of the chromosomes 9 and $22 \mathrm{t}(9 ; 22)(\mathrm{q} 34 ; \mathrm{q} 11)$ (1). This translocation creates two new genes, BCR-ABL on the 22q- (Ph chromosome) and the reciprocal ABL-BCR on 9q-. The BCR-ABL gene encodes for a $210-\mathrm{kD}$ protein with deregulated tyrosine kinase (TK) activity, resulting in CML. Small-molecule drugs, designed to interfere with BCR-ABL tyrosine kinase activation by competitive binding at the ATP-binding site have been developed. The first tyrosine kinase inhibitor (TKI), introduced into clinical practice, was imatinib mesylate (2). However, approximately $20-25 \%$ of patients initially treated with imatinib need alternative therapy, due to drug resistance, which is often caused by the appearance of clones expressing mutant forms of BCR-ABL. Imatinib has also shown to induce left ventricular contractile dysfunction (3). Dasatinib was the first, second-generation TKI, approved in the US and European Union for the treatment of

Correspondence to: Dr A.K.S. Bhujanga Rao, Natco Research Centre, B-13, Industrial Estate, Sanath Nagar, Hyderabad 500-018, India

E-mail: nrc@natcopharma.co.in

Key words: leukemia, chronic myeloid leukemia, imatinib, NRC-AN-019, BCR-ABL, tyrosine kinase inhibitor
CML patients with imatinib resistance or intolerance (4). This drug is a dual SRC-ABL kinase inhibitor, active in most clinically relevant BCR-ABL mutations, except the highly resistant T315I mutation. Other second-generation TKIs include nilotinib (5), bosutinib (6) and INNO 406 (7) which are showing promise as novel anti-CML agents but not promising for T315I mutants. Apart from TKIs, a promising group of molecules is inhibitors of Aurora family of serine-threonine kinases. One of these molecules, MK0457, has entered clinical trials, and initial reports indicate that this compound could be active in disease associated with T315I mutation (8). Thus, wide spectrum of new agents, with different modes of action, is currently in clinical development for CML and its long-term effectiveness against T315I mutants needs to be assessed. It is likely that combination therapy will be the best therapeutic strategy in the future. A recent computational study has suggested that the combination of two drugs is found to increase the probability of treatment success despite cross-resistance. Combining more than two drugs, however, does not provide further advantages (9). Several clinical trials revealed that imatinib mesylate induces hematologic remissions in up to $98 \%$ of patients, depending on the disease phenotype and phase (2,10-14). Crystallographic studies revealed that imatinib binds to the kinase domain of $\mathrm{c}-\mathrm{Abl}$ only when the domain adopts the inactive 'closed' conformation, which is required for optimal inhibition of the kinase activity $(15,16)$. However, despite the good hematological and cytogenic responses obtained, primary refractory disease and secondary resistance still occur with imatinib, particularly in patients with advanced phase disease (4). Although several mechanisms have been proposed to account for this resistance, including increased expression of BCR-ABL protein, amplification of the BCR-ABL gene and overexpression of the multi-drug resistance P-glycoprotein (17-19), point mutations in the BCR-ABL gene itself account for most cases of resistance. Based on crystallographic studies, we hypothesized that the potency and selectivity of imatinib mesylate could be improved by incorporating alternative binding groups to the $\mathrm{N}$-methyl piperazine moiety of the compound. This led to the development of NRC-AN-019, a high affinity phenyl amino pyrimidine based BCR-ABL inhibitor. NRC-AN-019 was developed using a rational drug design strategy based on the replacement of the methyl piperazinyl group of imatinib and optimization to improve potency against the ABL kinases. It retains the kinase specificity profile of imatinib, with inhibition of ABL kinases, ARG, Kit and PDGFR kinases. NRC-AN-019, in contrast to 
Table I. Scoring of imatinib and NRC-AN-019.

\begin{tabular}{lcccll}
\hline Molecule & PLP1 & PLP2 & DOCK & LIG2 & H-bonds \\
\hline $\begin{array}{l}\text { Reference } \\
\text { Imatinib }\end{array}$ & 160.16 & 154.12 & 148.15 & 8.16 & IEL360, ASP381, MET318, THR315, GLU286 \\
NRC-AN-019 & 166.46 & 158.82 & 147.15 & 8.30 & ASP381, MET318, THR315, GLU286 \\
\hline
\end{tabular}

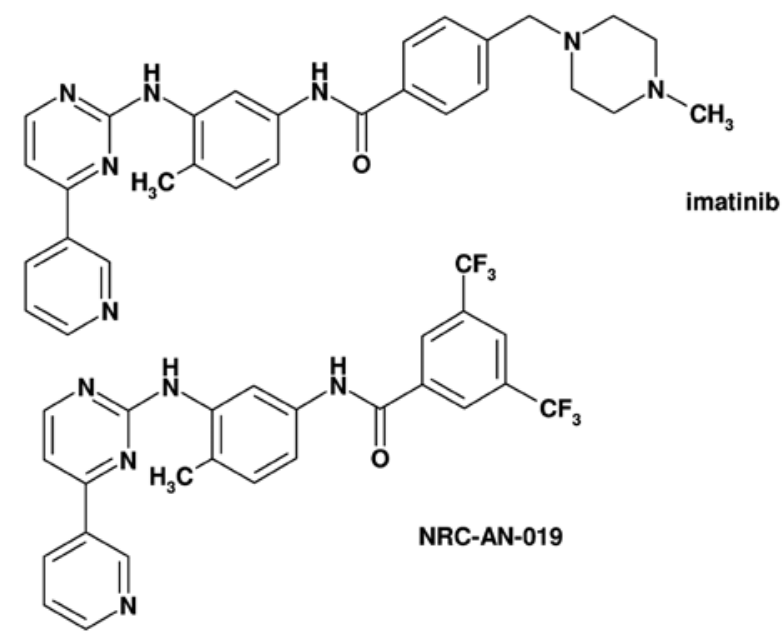

Figure 1. Structures of NRC-AN-019 and imatinib. The N-methyl piperazine moiety of imatinib is removed in AN019 and the phenyl ring is substituted in meta positions with two trifluoromethyl groups. This compact structure of AN-019 makes it better fit than imatinib.

dasatinib, binds the kinase domain of ABL kinases in the inactive conformation (Fig. 1). IUPAC name, 3,5- Bis(trifluoromethyl) -N-[4-methyl-3- [[4-(pyridin-3-yl)pyrimidin-2-yl] amino] phenyl] benzamide.

\section{Materials and methods}

Protein preparation. The crystal structure of BCR-ABL with its bound inhibitor imatinib was taken from the Protein Data Bank (PDB entry 1IEP) and was imported into Discovery Studio 2.1 (Accelrys Inc.) and the ligand was extracted to leave a cavity. The binding site prediction was based on receptor cavities. All $\mathrm{H}$-atoms were included.

Validation of the molecular docking method. To ensure that the ligand orientation and the position obtained from the docking studies were likely to represent valid and reasonable binding modes of the inhibitors, the ligand docking parameters had to be first validated for the crystal structure used PDB ID-1IEP and 2HYY. The ligand imatinib in the conformation found in the crystal structure, was extracted and docked back to the corresponding binding pocket, to determine the ability of ligand to reproduce the orientation and position of the inhibitor observed in the crystal structure.

Molecular docking studies. Molecular docking of NRC-AN-019 in the active site of BCR-ABL was carried out using modern docking engine LigandFit available with Discovery Studio 2.1
(Accelrys Inc.). NRC-AN-019 was manually docked into the binding site. As shown in docking diagrams, NRC-AN-019 was docked in the same binding site, by using docking procedure mentioned earlier. Fig. 5 depicts the docking model of ABL in complex with NRC-AN-019 and imatinib.

Scoring functions. The docked conformations were further scored using different scoring functions available with LigandFit. The Ligand algorithm uses an internal scoring function, Dock Score, to select and return dissimilar poses for each compound. Dock Score is a simple force field-based scoring function, which estimates the energy of interaction by summing the ligand/protein interaction energy and the internal energy of the ligand. CFF force field was used to resolve the van der Waals parameters for Dock Score. The top Dock Score pose was used for post-docking scoring. Scoring was performed using a set of scoring functions as implemented in LigandFit. These included LigScore1, LigScore2, PLP1, PLP2 and Dock Score available from the docking process. The putative 3D poses and score results were then stored as an SD file (Table I depicts the scoring of NRC-AN-019 and imatinib).

All the compounds reported herein were synthesized as follows. The synthesis of the derivatives $8(\mathrm{a}-\mathrm{g})$ is illustrated in Fig. 2A, $\alpha$-bromination of p-Toluic acid with $\mathrm{N}$-bromosuccinimide, subsequent hydrolysis with diluted hydrochloric acid followed by reaction with thionylchloride afforded acid chloride 4 . The benzoylation of the aniline 5 (13) with acid chloride 4 in chloroform solvent afforded the chloromethyl compound 6 . The condensation of chloromethyl compound with $7(\mathrm{a}-\mathrm{g})$ afforded the corresponding amides $8(\mathrm{a}-\mathrm{g})$. The synthesis of the derivatives 10(a-i) is illustrated in Fig. 2B. Condensation of acid chlorides of the commonly available acids 9a-I with the aniline 5 (13) in chloroform solvent afforded the corresponding amide compounds 10a-i. The anti-proliferative activities of all these compounds from two series $8(\mathrm{a}-\mathrm{g})$ and $10(\mathrm{a}-\mathrm{I})$ were evaluated in vitro against BCR-ABL positive cell line, D32p210 cell line (Fig. 3A and B). Briefly $5 \times 10^{3}$ cells were seeded per well in a 96-well plate and different concentrations of the compounds ranging from $1 \mathrm{nM}$ to $100 \mu \mathrm{M}$ were added in quadruplets. After incubating the cells with the compounds for the required time period ( $24 \mathrm{~h}$ ), $20 \mu \mathrm{l}$ of $5 \mathrm{mg} / \mathrm{ml}$ MTT (3-[4,5-dimethylthiazole2-yl]-2,5-diphenyl tetrazolium bromide) (13) was added (final concentration $100 \mu \mathrm{g} / \mu \mathrm{l}$ ) and incubated for additional $3 \mathrm{~h}$ at $37^{\circ} \mathrm{C}$ and $5 \% \mathrm{CO}_{2}$. After $3 \mathrm{~h}$, the formazone crystals were dissolved in lysis buffer $10 \%$ SDS, $5 \%$ isobutanol, $12 \mathrm{mmol} / 1 \mathrm{HCl}$ overnight at $37^{\circ} \mathrm{C}$. Absorbance was measured on ELISA reader at dual wave length of 570-630 nm. NRC-AN-019 and imatinib mesylate were dissolved in dimethylsulfoxide (DMSO). The stock solution was kept at $-20^{\circ} \mathrm{C}$. Imatinib mesylate was used as a control compound. 
A<smiles>Cc1ccc(C(=O)O)cc1</smiles>

p-Toluic acid<smiles>CC(=O)Nc1ccc(C)c(Nc2nccc(-c3cccnc3)n2)c1</smiles><smiles>CCC</smiles><smiles>O=C(Cl)c1ccc(CCl)cc1</smiles>

4 $7 \mathbf{a}, \mathbf{8} \mathbf{a}: \mathbf{R 1}=$<smiles>C1COCCN1</smiles><smiles>C1CCNCC1</smiles><smiles>c1c[nH]cn1</smiles>

7d, 8d : R1 =<smiles>c1nc[nH]n1</smiles>

$7 e, 8 e: R 1=$<smiles>CC(C)NC(C)C</smiles>

7f , 8f : R1 = $\mathrm{NH}\left(\mathrm{CH}_{3}\right)_{2}$

$7 g, 8 g: \mathbf{R 1}=$<smiles>CCCCNCCC</smiles>

Figure 2. (A) Scheme showing reagents and conditions for synthesis of candidate compounds a-NBS, dibenzoyl peroxide, b- $\mathrm{HCl}$ reflux, c-SOCl2 reflux, d-trimethylamine, chloroform, e- a to g, DMF.

Cell line and culture conditions. The human chronic myeloid leukemia K562 cells (American Type Culture Collection, Manassas, VA) were maintained in RPMI-1640 medium (Hyclone, Logan, UT) supplemented with $10 \%$ fetal bovine serum (Hyclone), penicillin $(100 \mathrm{U} / \mathrm{ml})$ and streptomycin (100 mg/ml). K562 cells stably transfected with luciferace expressing plasmids were also used. Murine $\mathrm{Ba} / \mathrm{F} 3$ cells, which stably express BCR-ABL with point-mutated kinase domain (T315I), were kindly provided by Dr Brian Druker (Oregan Health and Science University, Portland, OR) and cultured under the conditions described above.
Animal studies. For in vivo experiments involving animals due permission for conduct of these experiments was obtained, from the relevant ethics committees. Nude mice were obtained from Harlan Laboratories of North America and housed as per recommended guidelines in a sterile germ-free environment.

Therapeutic activity. Establishment of anti-CML activity of NRC-AN-019 in nude mice implanted with K562 or K562-luc cells was done as follows. To determine anti-CML activity of NRC-AN-019, K562 (luc) cells were implanted into nude mice via tail vein injection and treated with various concentra- 
B<smiles>Cc1ccc(N)cc1Nc1nccc(-c2cccnc2)n1</smiles><smiles>CCCC(=O)Nc1ccc(C)c(Nc2nccc(-c3cccnc3)n2)c1</smiles>
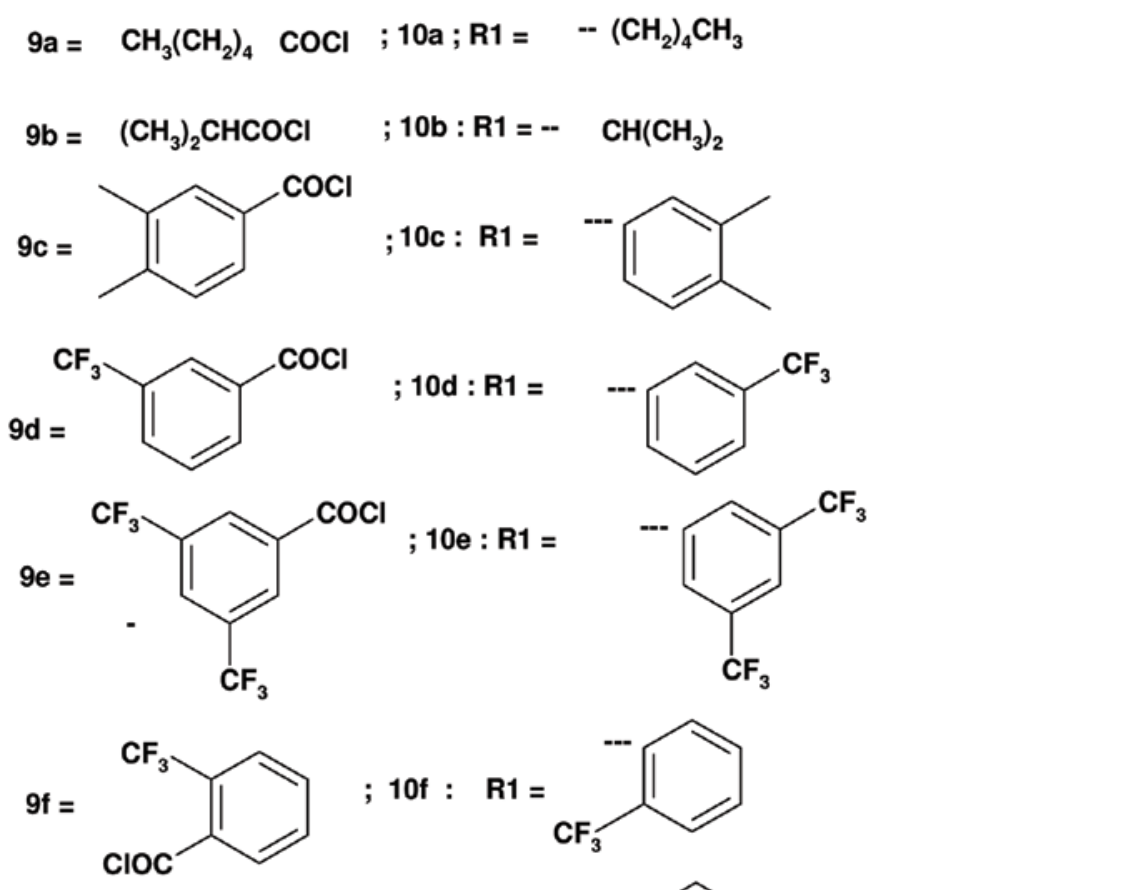

$9 g=$<smiles>O=C(OC(=O)C(F)(F)F)c1cccnc1C(F)(F)F</smiles>

; $10 \mathrm{~g}: \mathbf{R 1}=$<smiles>Cc1cccnc1C(F)(F)F</smiles>

$9 \mathrm{~h}=$<smiles>O=C(O)c1cccc(S(F)(F)(F)(F)F)c1</smiles>

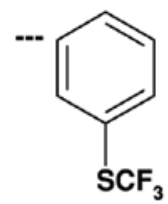

$9 \mathbf{i}=$

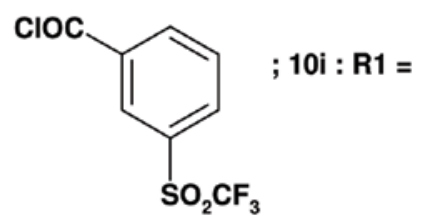<smiles>Cc1cccc(S(=O)(=O)OC(F)(F)F)c1</smiles>

Figure 2. Continued. (B) Scheme showing reagent conditions for synthesis of candidate compounds 9a-i a-chloroform and triethyl amine.

tions of NRC-AN-019 or imatinib (5, 10, 20 and $40 \mathrm{mg} / \mathrm{kg}$ ). Stock solutions of imatinib and NRC-AN-019 were made at a concentration of $100 \mu \mathrm{g} / \mu \mathrm{l}$ in DMSO. Mice were injected via an intraperitoneal (IP) route at the above-mentioned dosage. Average weight of nude mice was determined to be $30 \pm 3 \mathrm{~g}$.
Three microliters of the stock solutions was diluted to $100 \mu \mathrm{l}$ with sterile water just prior to IP injections. IP injections were carried out daily from day 15 post-implantation to day 48 . K562 luciferase-implanted nude mice were scanned using the Xenogen IVIS ${ }^{\circledast}$ image system after IP injections of luciferin 


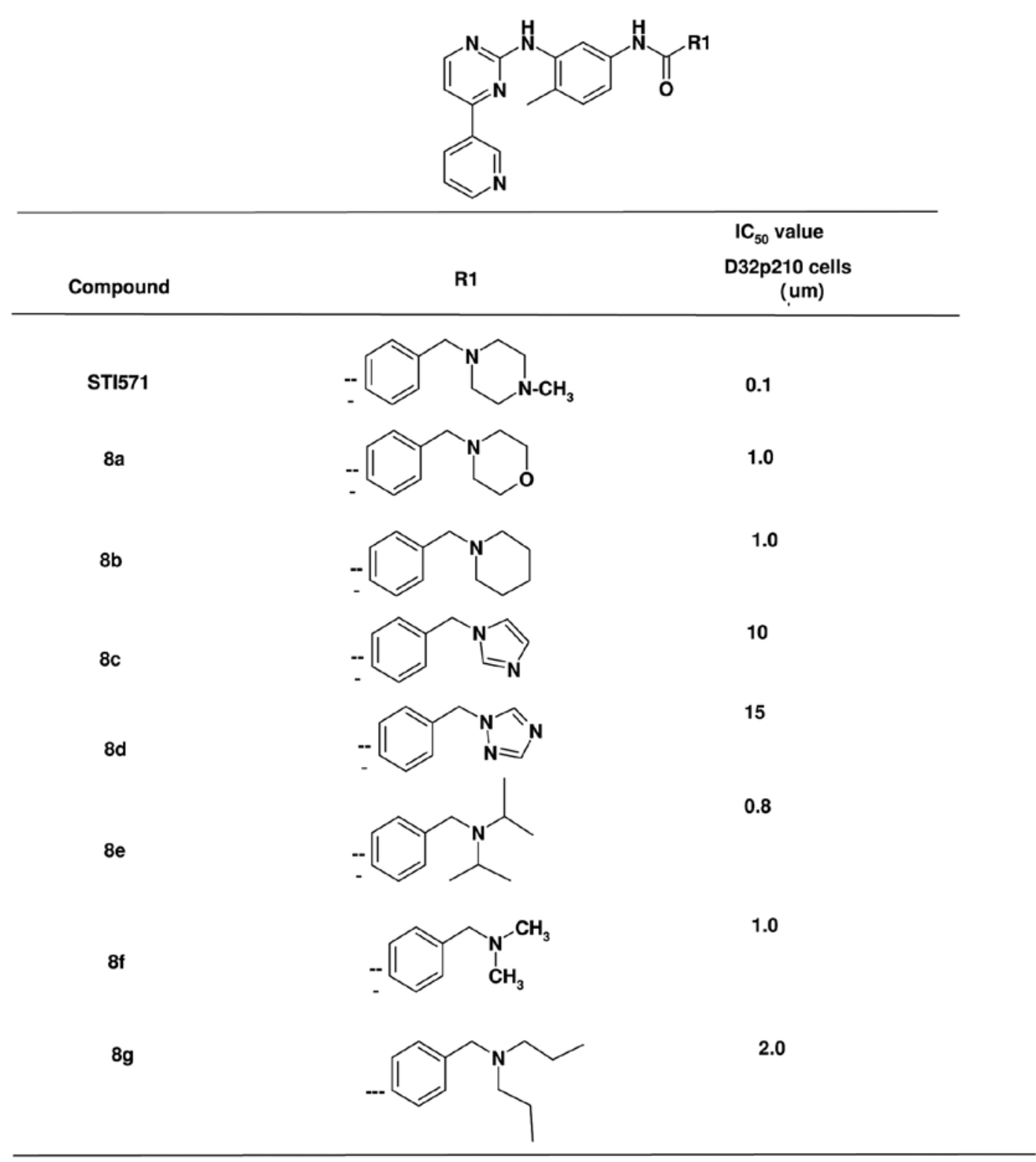

Figure 3. (A) Antiproliferative activity of the compounds prepared by following Fig. 2A.

(50 $\mu \mathrm{g} /$ mouse). Blood was drawn from the tail vein every 6 th day and blast cell count determined to determine the leukemia growth index (LGI). Leukemia growth index was determined using the formula:

$$
L G I(\%)=\left(\frac{\left(V_{c}-V_{t}\right)}{V_{c}}\right) 100
$$

$\mathrm{V}_{\mathrm{c}}$ is mean blast cell number (K562) per ml of blood in control animals at a certain time of measurement, and $\mathrm{V}_{\mathrm{t}}$ is mean blast cell number per $\mathrm{ml}$ of blood in test animals at a certain time of measurement. Blood was drawn from the mice (treated and untreated) on the 6th day post-K562 cell implantation and followed every 6-day interval for 48 days.

Determination of drug temporal penetrance $\left(T_{p}\right)$. To determine the temporal penetrance of NRC-AN-019 and imatinib, nude mice were implanted with K562-luc cells. The animals were imaged at 6-day intervals post-transplantation. Drug treatment (NRC-AN-019 $20 \mathrm{mg} / \mathrm{kg}$ and imatinib $10 \mathrm{mg} / \mathrm{kg}$ ) was initiated 15 days post-implantation by daily IP injections. Drug treatment was withdrawn on day 35 and animals were imaged till day 45 . The temporal penetrance was calculated applying the following equation:

$$
\frac{(P / n)_{a}-(P / n)_{\text {ter }}}{(P / n)_{a}-(P / n)_{b}}=T_{p}
$$

Where P, photon count at days ' $a$ ', 'ter' or 'b' where 'a' is the day when drug treatment was stopped; 'ter' is the day when the experiment was terminated; and ' $b$ ' is the day where $\mathrm{P}$ is minimum after day ' $\mathrm{a}$ ' but before day 'ter'. $\mathrm{N}$, number 
B

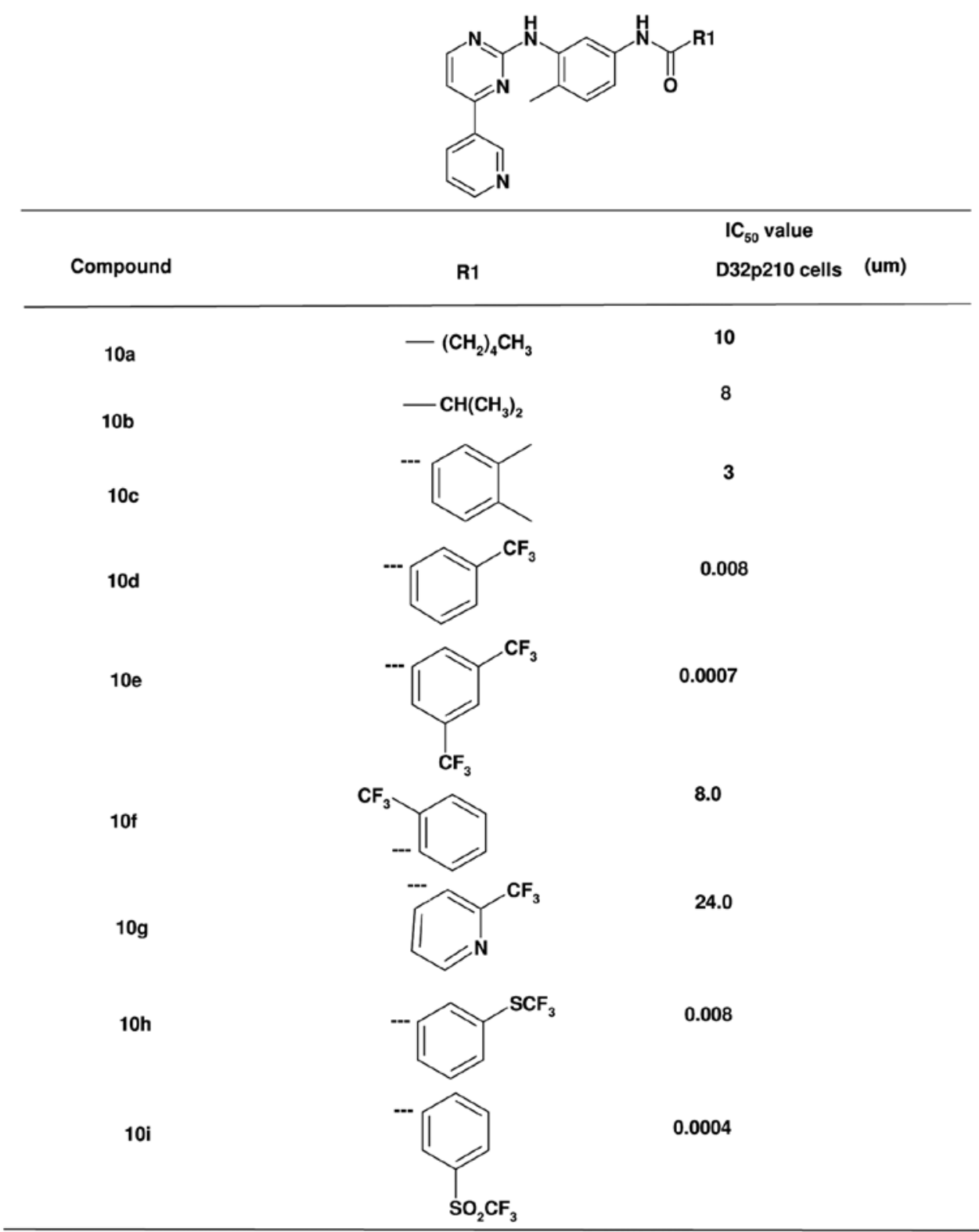

Figure 3. Continued. (B) Antiproliferative activity of the compounds prepared by following Fig. 2B.

of animals alive when $\mathrm{P}$ was measured. The larger the value indicates greater effectiveness of the drug after stopping drug treatment i.e., the penetrance of the drug over time.

Determination of drug effectiveness $\left(D_{e}\right)$. Drug effectiveness was determined using the equation below for animals implanted with K562 (luc) cells:

$$
\left\{\frac{\left[\sum \text { alive } / \sum l u c\right]-\left[\sum_{c} \text { alive } / \sum_{c} l u c\right]}{\sum_{c \text {-initial }} \text { alive }}\right\} 100=D_{e}
$$

Where, Salive, total number of mice alive per concentration at the end of experiment times photon count; $\sum l u c$, total number of mice alive showing luciferace expression per concentration at end of experiment; $c$, control untreated animals times photon count; and $\sum_{c \text {-initial }}$, the initial number of animals in control at start of experiment times photon count. The results are represented as percent drug effectiveness.

Animal survival study. Nude mice were implanted with K562-luc cells. Animals were treated as mentioned in Table II every day for 55 days; treatments were initiated 15 days post-implantation. Luc images were obtained at regular intervals and blood samples were obtained every 6th day and graphically represented. 
A
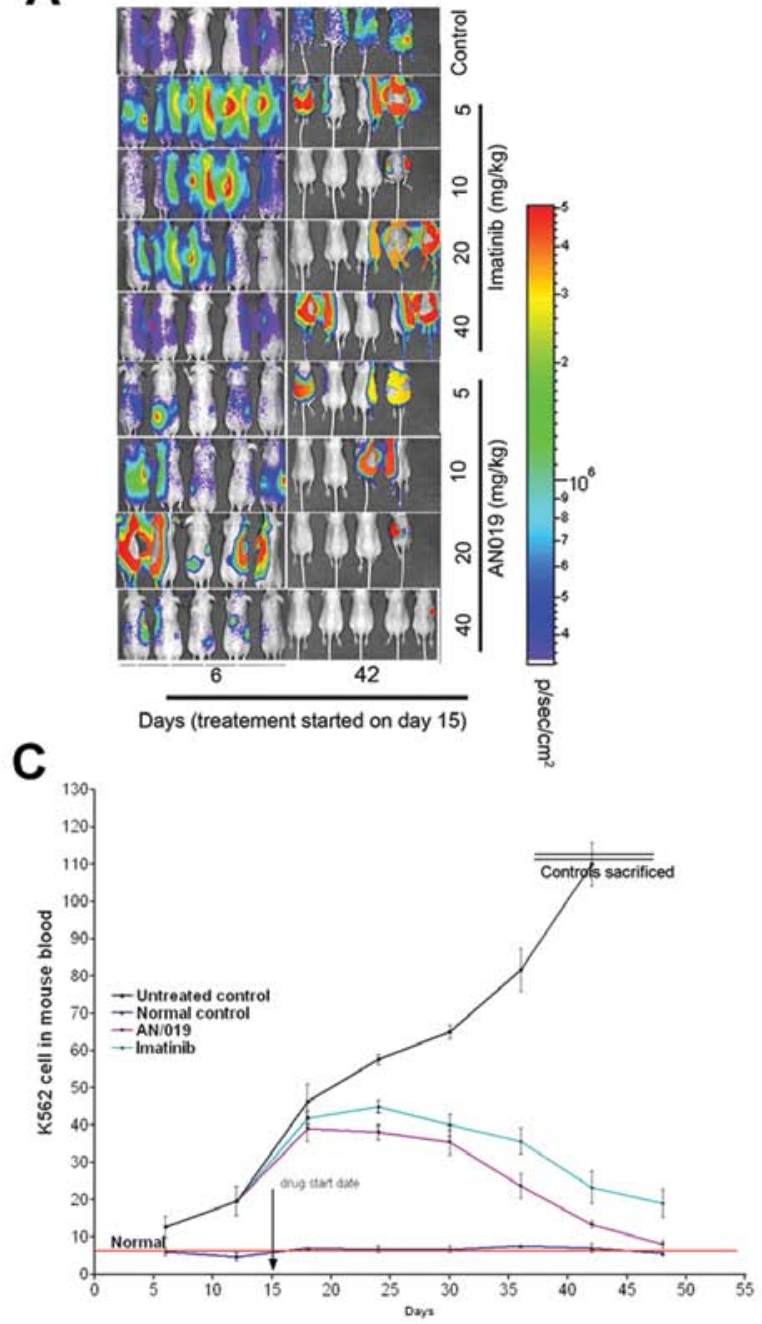

B

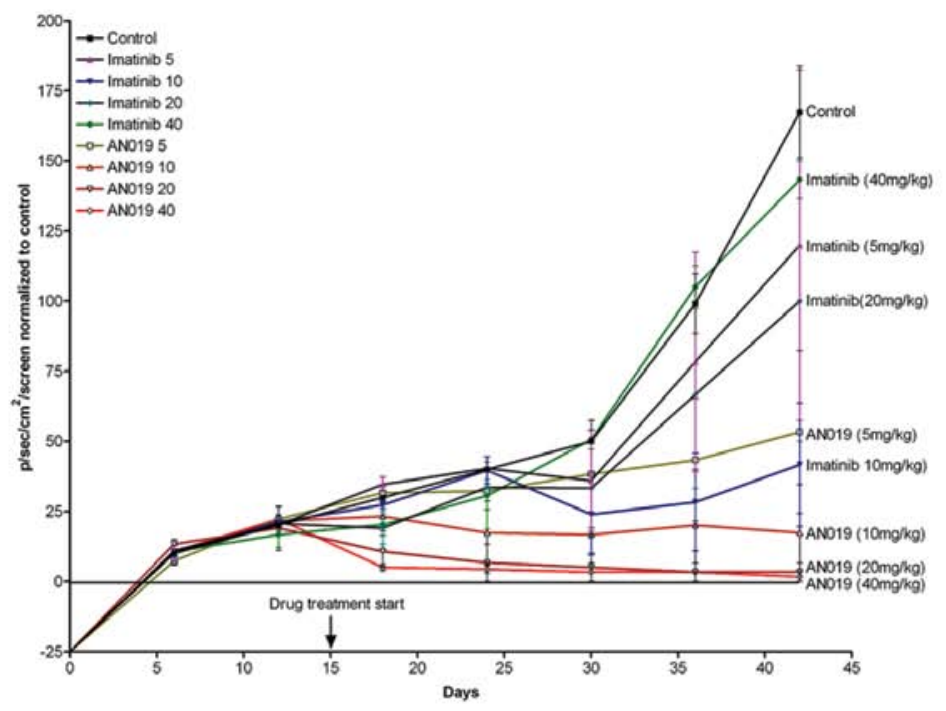

D

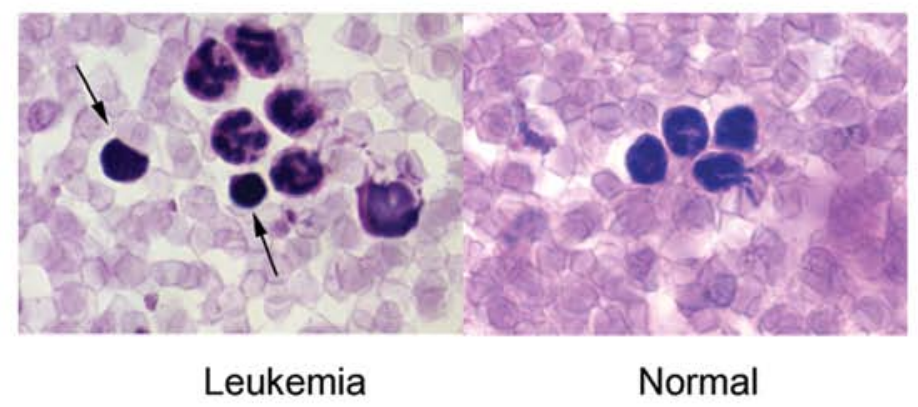

Figure 4. Nude mice were implanted with K562-luc cells and treated with varying concentrations of NRC-AN-019 or imatinib. (A) Luciferace images were obtained every 6th day to monitor progression of leukemic symptoms. (B) Photon counts obtained from the K562-luc-implanted mice were graphically plotted normalized to controls to determine the effect of NRC-AN-019 and imatinib treatments. (C) To correlate the leukemic symptoms from the luciferace expressions blast cell counts were determined in mice treated with $20 \mathrm{mg} / \mathrm{kg}$ of NRC-AN-019 or imatinib and plotted as blast cells per $100 \mu \mathrm{l}$. Panel (D) showed morphology of leukemic vs. normal mouse blood.

Table II. Animal survival study.

\begin{tabular}{lccc}
\hline Drug & Dose mg/kg & Animals & Cell line \\
\hline Imatinib & 20 & 10 & K562luc \\
NRC-AN-019 & 20 & 10 & K562luc \\
Control & 0 & 10 & K562luc \\
\hline
\end{tabular}

Determination of anti-CML activity of NRC-AN-019 against Baf3 (T315I) imatinib resistant cells. Nude mice were implanted with Baf3 (T315I) or Baf3 WT cell via the tail vein and allowed to grow for 15 days. Mice were then treated with NRC-AN-019 or imatinib $(20 \mathrm{mg} / \mathrm{kg})$ every day by intraperitoneal injections and blood from tail vein or the femoral vein was taken every 6th day until day 42 and blast cell counts determined.

\section{Results}

NRC-AN-019 has greater temporal penetrance and effectiveness than imatinib. By applying the equation (equation 2) for temporal penetrance, it was determined that $T_{p}$ values for $\mathrm{NRC}-\mathrm{AN}-019=2.0$ and imatinib $=0.8$, indicating that NRC-AN019 had superior activity and fared better than imatinib in terms of persistence over time. To determine the drug effectiveness over varying concentrations we observed that NRC-AN-019 was more effective with increasing concentrations when compared to imatinib (Table III).

Intraperitoneal injections of NRC-AN-019 caused regression of leukemia in nude mice. Mice implanted with K562-luc cells were scanned using the Xenogen IVIS ${ }^{\circledR}$ imaging system to determine progression of leukemia. We observed that mice treated with NRC-AN-019 showed decreased leukemia symptoms in a dose dependent manner when compared to 

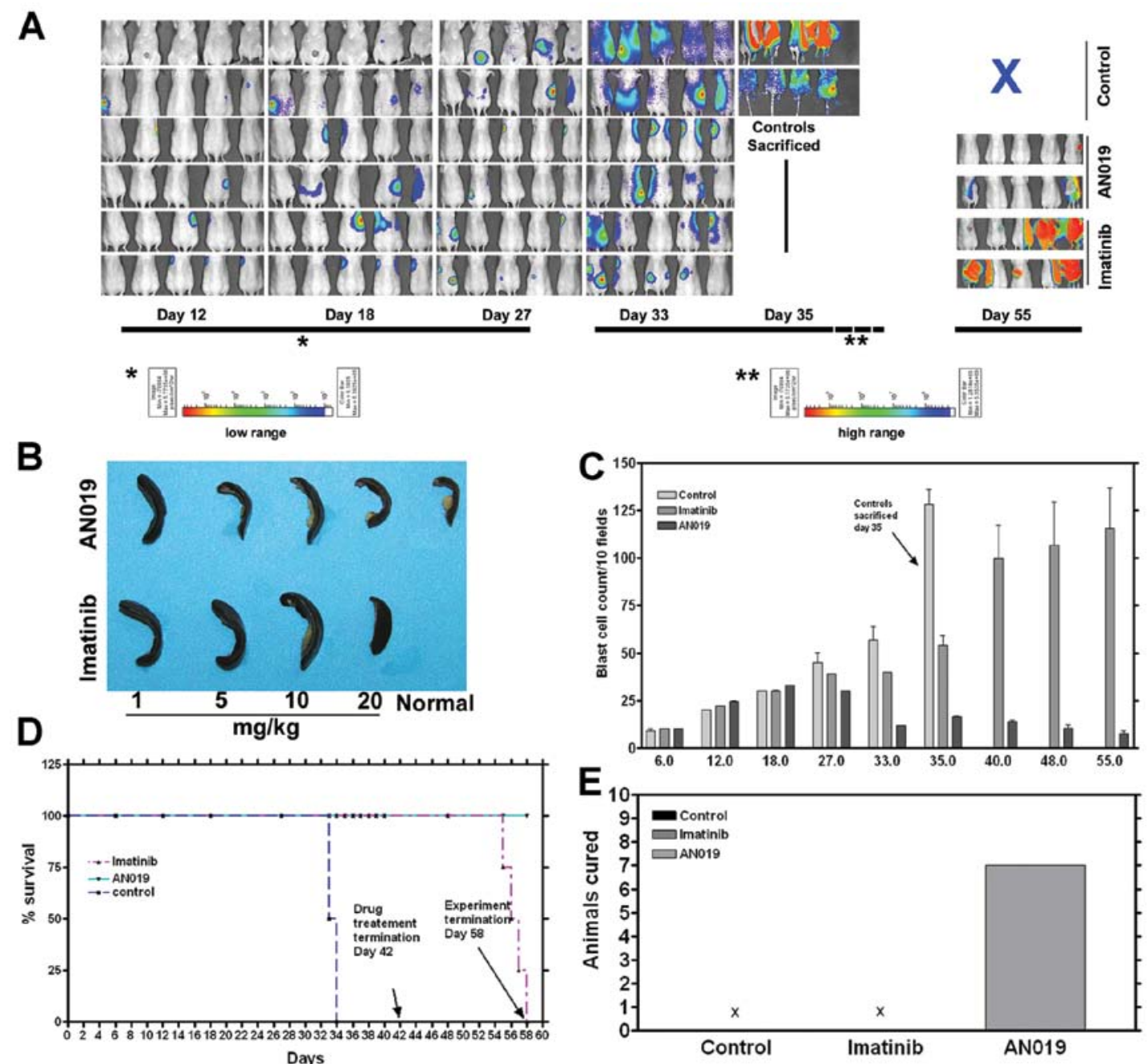

Figure 5. To determine the survival of leukemic mice after NRC-AN-019 or imatinib treatment, mice were implanted with K562-luc cells and treated as indicated in Materials and methods. (A) Luciferace imaging was done from day 12 to day 55. (B) To also determine whether mice showed splenic enlargement, spleens were harvested from mice treated with varying concentrations of NRC-AN-019 or imatinib and image to determine relative size. (C) From the mice used for the survival study blood was periodically drawn and blast cell count determined. (D) A Kaplan-Meier curve was plotted to determine percent survival of mice. (E) Graphical representations of number of cured mice after 55 days. Mice were treated with NRC-AN-019 or imatinib (20 mg/kg see Materials and methods).

Table III. Temporal penetrance and effectiveness of NRC-AN-019 and imatinib.

\begin{tabular}{lcccr}
\hline Drug & $\mathrm{mg} / \mathrm{kg}$ & $10 \mathrm{mg} / \mathrm{kg}$ & $20 \mathrm{mg} / \mathrm{kg}$ & $40 \mathrm{mg} / \mathrm{kg}$ \\
\hline Imatinib mesylate & 10.00 days & 32.00 days & 13.33 days & 8.00 days \\
NRC-AN-019 & 25.82 days & 78.20 days & 83.40 days & 85.20 days \\
\hline
\end{tabular}

imatinib-treated mice (Fig. 4A). Quantitative analysis of photon count revealed that mice treated with $5 \mathrm{mg} / \mathrm{kg}$ of NRC-AN-019 showed decreased leukemia comparable to mice treated with $10 \mathrm{mg} / \mathrm{kg}$ of imatinib. Increase in dose of imatinib treatments (20 and $40 \mathrm{mg} / \mathrm{kg}$ ) did not show a dose dependent response as observed in NRC-AN-019 treated mice (Fig. 4B). Blood smears taken every 6th day post-implantation indicated an increase in LGI in untreated controls when compared to imatinib and NRC-AN-019 treatments. From the 15th day post-implantation, mice were given intraperitoneal injections of NRC-AN-019 or imatinib at a dosage of $10 \mathrm{mg} / \mathrm{kg}$ body weight. Imatinib-treated mice lagged NRC-AN-019-treated mice at every data point.
On day 48 LGI of imatinib-treated mice was determined to be $20 \pm 5$, whereas LGI of NRC-AN-019-treated mice was found to be $10 \pm 2$ with an almost normal cell count (Fig. 4C and D). The study shows the superiority of NRC-AN-019 over imatinib in leukemic regression, particularly after 24 days of treatment.

Survival study. This study was conducted to determine the survival benefit of NRC-AN-019 in leukemic mice. Fifteen days post-implantation, we treated animals with NRC-AN-019 and imatinib for 55 days and luciferace images obtained every 6 th day to determine leukemia progression. Control animals developed leukemia and mortality had occurred on days 34 


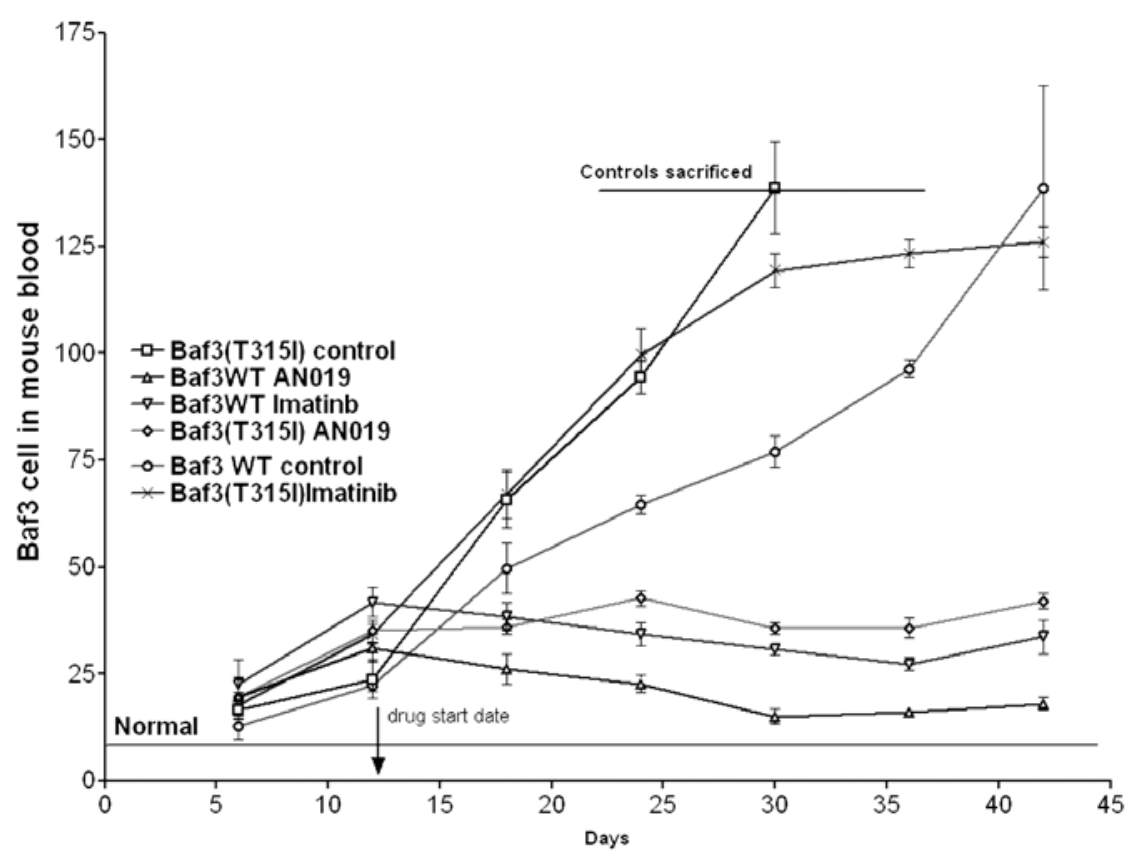

Figure 6. To determine whether NRC-AN-019 can suppress imatinib resistant leukemia, mice were implanted with imatinib resistant murine Baf3 (T315I) cells and treated with $20 \mathrm{mg} / \mathrm{kg}$ of NRC-AN-019 or imatinib. Blood was drawn periodically and blast cell count determined and graphically represented.

and 35 . As per ethics committee regulations we were advised to sacrifice the remaining 8 animals on day 35 , drug treatment was withdrawn on day 42 post-implantation and survival of animals determined by luciferace imaging (Fig. 5A). Blood smears were taken from the leukemic animals at regular intervals, it was observed that the blast cell count in controls animals reached a maximum by day 35 and subsequently they were sacrificed. However, animals treated with NRC-AN-019 continued to show regression in blast cell count even after withdrawal of drug treatment after day 42. Blast cell counts almost reached normal levels at day 58. Animals treated with imatinib showed a decrease in blast cell count when compared to controls, whereas after withdrawal of imatinib blast cell count continued to increase (Fig. 5C). Animals treated with NRC-AN-019 did not show any mortality and 7 of the 10 animals showed absence of leukemic symptoms. Animals treated with imatinib showed reoccurrences of leukemic symptoms after treatment withdrawal and showed mortality on days 55, 56, 57 and 58; the surviving animals did show presence of leukemic symptoms (Fig. 5D).

NRC-AN-019 injections of nude mice implanted with K562 cells did not show splenic enlargement. Nude mice implanted with K562 cells were treated with imatinib or NRC-AN-019 via IP injections. Mice were sacrificed after a decrease in LGI was observed and spleens harvested. Spleenic enlargement was observed by gross observation and it was determined that control mice showed enlarged spleen indicative of K562 cellular localization. NRC-AN-019-treated animals did not show any spleenic enlargement whereas imatinib-treated mice showed slight spleenic enlargement (Fig. 5B). Imatinib-treated mice also showed significant reduction in LGI but lagged NRC-AN-019-treated mice. From these results, it is evident that
NRC-AN-019 could be a promising therapeutic drug for the treatment of chronic myeloid leukemia.

Mice implanted with imatinib resistant Baf3T315I cells respond to NRC-AN-019. It was observed that in Baf3Wt-implanted mice, progressive decrease in blast cell count was observed in both NRC-AN-019 and imatinib treatment conditions. Baf3T315I did not respond well to imatinib treatments and showed a progressive increase in blast cell count similar to controls but showed a plateau after day 30 indicating a slight decrease in leukemia. NRC-AN-019 treatments were able to reduce leukemia symptoms significantly in both Baf $3 \mathrm{Wt}$ and Baf3 (T315I) imatinib resistant leukemic mice. Overall intraperitoneal treatments of NRC-AN-019 were significantly better at decreasing blast cell count in all Baf3-implanted mice (Fig. 6).

\section{Discussion}

Compared with imatinib, dasatinib was developed from a completely different chemical scaffold. In contrast, NRC-AN-019 is the result of rational modifications to the imatinib molecule.

Crystal structure analysis had revealed that much of the binding energy of imatinib was consumed by energetically unfavorable hydrogen bond between the $\mathrm{N}$-methyl piperazine moiety and $\mathrm{Ile}^{360}$ of ABL (Fig. 7A). Replacement of the piperazine ring with two meta substitituted trifluoromethyl groups eliminated these two hydrogen bonds. The result is a better topological fit and a molecule that inhibits ABL with 140-fold increased potency compared with the parental compound (Fig. 7B).

The most potent compounds in Table II are $10 \mathrm{e}$ (NRC-AN-019), 10h and 10i (AN-024). We selected 10e with 
A.

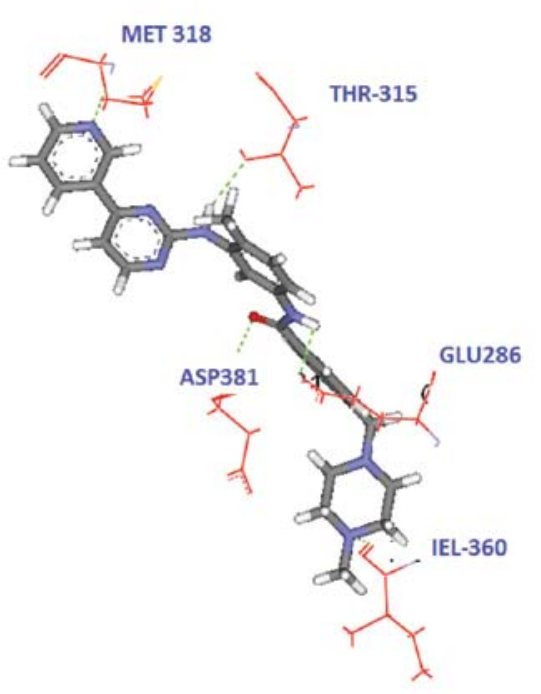

B.

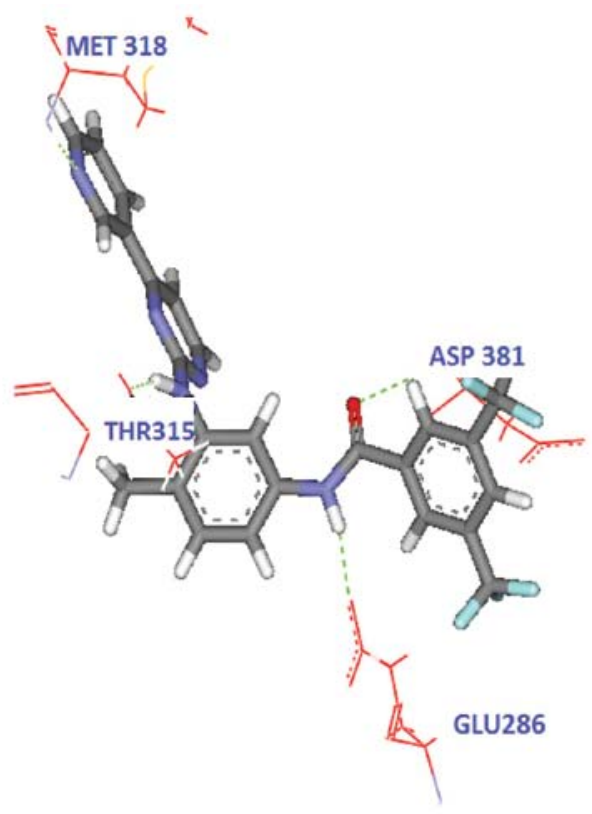

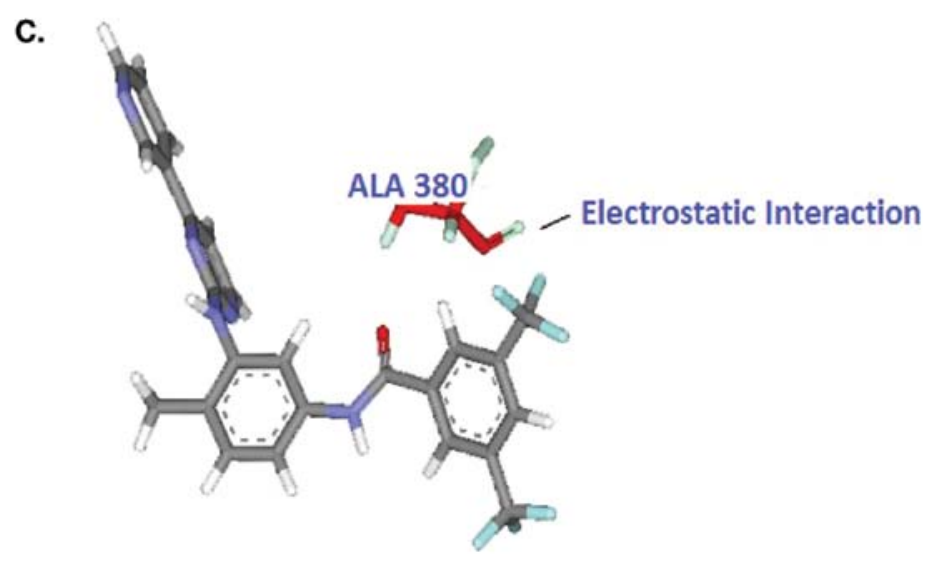

Figure 7. Interaction of imatinib and NRC-AN-019 with BCR-ABL. The reduced requirement for hydrogen bonding with NRC-AN-019 compared to imatinib, allows for a tighter fit into the ATP. (A) Hydrogen bonds (H-bonds) of imatinib represented with green dots (5 H-bonds). (B) H-bonds of NRC-AN-019 represented with green dots (4 H-bonds only). (C) Figure shows electrostatic interaction of NRC-AN-019 with Alanine-380.

$\mathrm{IC}_{50}$ of $0.7 \mathrm{nM}$ that is about 140 -fold more potent than imatinib as a promising candidate for development judging its overall characteristics. NRC-AN-019 binds only to the inactive conformation of Bcr-Abl, with P-loop folding over the ATP-binding site, and the activation-loop blocking the substrate binding site, to disrupt the ATP-phosphate-binding site and inhibit the catalytic activity of the enzyme. NRC-AN-019 makes 4 hydrogen-bond interactions with the ABL kinase domain, involving the pyridyl-N and the backbone-NH of Met318, the aniline-NH and the side chain hydroxyl of Thr315, the amido-NH and side chain carboxylate of Glu286, as well as the amido- $\mathrm{C}=\mathrm{O}$ of Asp381 and a fluorine atom in the trifluoromethyl group of NRC-AN-019. This close interaction made changes in the core of imatinib prohibitive. The improvement in binding affinity for BCR-ABL maintains the ability to inhibit not only Kit but also PDGFR. NRC-AN-019 has a better fit to the DFG-out pocket than imatinib, which probably accounts largely for its increased affinity. There is also a weak electrostatic interaction between fluorine from the trifluoro- methyl group of NRC-AN-019 (10e, Fig. 2B) and the polarized $\mathrm{C}$ atom of the Ala380 carbonyl (Fig. 7C).

Dose response studies indicated that with increasing dose of NRC-AN-019 the response rate also increases, whereas in case of imatinib, increasing dose did not induce a dose dependent response. In fact a lower concentration of imatinib was observed to be more effective than higher concentration. From the blast cell count of mice treated with $10 \mathrm{mg} / \mathrm{kg}$ of NRC-AN019 or imatinib we observed a progressive decrease blast cell count over time, with NRC-AN-019-treated mice doing better than imatinib-treated mice. This correlates well with our drug effectiveness studies where we observed that at $5 \mathrm{mg} / \mathrm{kg}$ NRC-AN-019 was twice as effective as imatinib but at $40 \mathrm{mg} /$ kg NRC-AN-019 was at least 10 times as effective as imatinib. From the survival analysis in which we used K562-implanted mice and treated with $20 \mathrm{mg} / \mathrm{kg}$ of NRC-AN-019 or imatinib, we observed that control mice showed full blown leukemia symptoms by day 35 after which they were sacrificed. By day 55 most of the NRC-AN-019-treated mice were 
leukemia-free, whereas imatinib-treated mice still showed leukemia symptoms. This indicates that even after withdrawal of treatment mice continue to show decrease in leukemia symptoms, whereas withdrawal of imatinib causes recurrence of leukemia. This also correlates with our temporal penetrance analysis where we observed that NRC-AN-019 was at least twice as effective as imatinib after withdrawal of treatment. A hallmark of leukemia is the enlargement of the spleen, we observed that NRC-AN-019-treated mice did not show spleenic enlargement whereas imatinib-treated mice showed spleenic enlargement and was inversely proportional to dose. Survival analysis clearly indicates that NRC-AN-019 was more beneficial than imatinib. Overall NRC-AN-019 was able to suppress leukemia in at least $90 \%$ of the animals treated, when compared to imatinib treatments. Finally, the use of imatinib resistant Baf3 (T315I) cells showed that NRC-AN-019 was able to overcome the imatinib resistance and show a good positive curative effect even at a dose of $20 \mathrm{mg} / \mathrm{kg}$.

In conclusion, we have designed and synthesized some phenyl amino pyrimidine derivatives and evaluated them as BCR-ABL tyrosine kinase inhibitors. We identified clinical candidate 10e (NRC-AN-019) as a highly potent BCR-ABL kinase inhibitor. We conclude that NRC-AN-019 holds great potential for therapeutic use in the treatment of $\mathrm{Ph}^{+}$leukemias, breast cancers and brain cancers. Our studies show that NRC-AN-019 is superior to imatinib and is highly effective in eliminating large number of leukemic cells in vivo. We expect NRC-AN-019 to be a potential candidate for proceeding to clinical trials.

\section{Acknowledgements}

The authors thank the management of Natco Pharma Limited for encouragement and support. The technical help from the Department of Pharmacology and Cancer Biology, University of Illinois at Peoria is gratefully acknowledged.

\section{References}

1. Rowley JD: Letter: A new consistent chromosomal abnormality in chronic myelogenous leukaemia identified by quinacrine fluorescence and Giemsa staining. Nature 243: 290-293, 1973.

2. Buchdunger E, Zimmermann J, Mett $\mathrm{H}$, et al: Inhibition of the Abl protein-tyrosine kinase in vitro and in vivo by a 2-phenylaminopyrimidine derivative. Cancer Res 56: 100-104, 1996
3. Risto K: Cardiotoxicity of the cancer therapeutic agent imatinib mesylate. Nat Med 12: 908-916, 2006.

4. Shah NP, Tran C, Lee FY, Chen P, Norris D and Sawyers CL: Overriding imatinib resistance with a novel ABL kinase inhibitor. Science 305: 399-401, 2004.

5. Weisberg E and Griffin JD: Mechanism of resistance to the ABL tyrosine kinase inhibitor STI571 in BCR/ABL-transformed hematopoietic cell lines. Blood 95: 3498-3505, 2000.

6. Vultur A, Buettner R, Kowolik C, et al: SKI-606 (bosutinib), a novel Src kinase inhibitor, suppresses migration and invasion of human breast cancer cells. Mol Cancer Ther 7: 1185-1194, 2008.

7. Kimura S, Naito H, Segawa $\mathrm{H}$, et al: NS-187, a potent and selective dual Bcr-Abl/Lyn tyrosine kinase inhibitor, is a novel agent for imatinib-resistant leukemia. Blood 106: 3948-3954, 2005.

8. Maekawa T, Ashihara E and Kimura S: The Bcr-Abl tyrosine kinase inhibitor imatinib and promising new agents against Philadelphia chromosome-positive leukemias. Int J Clin Oncol 12: 327-340, 2007.

9. Komarova NL, Katouli AA and Wodarz D: Combination of two but not three current targeted drugs can improve therapy of chronic myeloid leukemia. PLoS One 4: e4423, 2009.

10. Druker BJ, Sawyers CL, Kantarjian H, et al: Activity of a specific inhibitor of the BCR-ABL tyrosine kinase in the blast crisis of chronic myeloid leukemia and acute lymphoblastic leukemia with the Philadelphia chromosome. N Engl J Med 344: 1038-1042, 2001.

11. Druker BJ, Talpaz M, Resta DJ, et al: Efficacy and safety of a specific inhibitor of the BCR-ABL tyrosine kinase in chronic myeloid leukemia. N Engl J Med 344: 1031-1037, 2001.

12. Kantarjian H, Sawyers C, Hochhaus A, et al: Hematologic and cytogenetic responses to imatinib mesylate in chronic myelogenous leukemia. N Engl J Med 346: 645-652, 2002.

13. Talpaz M, Silver RT, Druker BJ, et al: Imatinib induces durable hematologic and cytogenetic responses in patients with accelerated phase chronic myeloid leukemia: results of a phase 2 study. Blood 99: 1928-1937, 2002.

14. Nowell PC: A minute chromosome in human chronic granulocytic leukemia. Science 132: 1497-150, 2012.

15. Schindler T, Bornmann W, Pellicena P, Miller WT, Clarkson B and Kuriyan J: Structural mechanism for STI-571 inhibition of abelson tyrosine kinase. Science 289: 1938-1942, 2000.

16. Nagar B, Bornmann WG, Pellicena P, et al: Crystal structures of the kinase domain of c-Abl in complex with the small molecule inhibitors PD173955 and imatinib (STI-571). Cancer Res 62: 4236-4243, 2002.

17. Tipping AJ, Mahon FX, Lagarde V, Goldman JM and Melo JV: Restoration of sensitivity to STI571 in STI571-resistant chronic myeloid leukemia cells. Blood 98: 3864-3867, 2001.

18. Mahon FX, Deininger MW, Schultheis B, et al: Selection and characterization of BCR-ABL positive cell lines with differential sensitivity to the tyrosine kinase inhibitor STI571: diverse mechanisms of resistance. Blood 96: 1070-1079, 2000.

19. le Coutre P, Tassi E, Varella-Garcia M, et al: Induction of resistance to the Abelson inhibitor STI571 in human leukemic cells through gene amplification. Blood 95: 1758-1766, 2000. 